\section{Percepción de la población con respecto a medicamentos genéricos frente a los de marca en hospitales del Perú}

\author{
The population's perceptions of generic drugs \\ compared to original brand-name drugs in \\ Peruvian hospitals
}

\section{Percepção da população a respeito dos medicamentos genéricos frente aos de marca em hospitais do Peru}

Giuston Mendoza-Chuctaya 1 Wildor Samir Cubas-Llalle 2 Christian R. Mejia 3 Jorge Emerson Chachaima-Mar 4 Reneé Montesinos-Segura 1 Laura R. Arce-Villalobos 5 John Carlos Mamani-Cruz 6

\section{Resumen}

El objetivo del estudio fue conocer la percepción de la población con respecto a medicamentos genéricos, frente a los medicamentos de marca, en hospitales del Perú. Participaron del estudio 4.914 personas mayores de 18 años, de 13 ciudades del Perú; clasificándolas en Lima, grandes y pequeñas ciudades. Se exploraron características socioeconómicas, demográficas y de percepción de medicamentos genéricos, en comparación con los medicamentos de marca. Determinando las asociaciones para cada cruce de variables, se calcularon razones de prevalencias (RP) y sus intervalos del 95\% de confianza (IC95\%), usando regresiones de Poisson crudas y ajustadas con varianza robusta con Stata 14.0. De los 4.914 participantes, el 46,7\% estaban de acuerdo con que los medicamentos genéricos son menos eficaces que los medicamentos de marca, el 49,3\% ha recomendado o recomendaría a otras personas el uso de medicamentos genéricos, además, el análisis multivariado encontró que las personas que tenían un ingreso económico menor a PEN 1.000 estaban predispuestas a recomendar un medicamento genérico $(R P=1,36$; IC95\%: 1,14-1,63). Los resultados ponen en manifiesto que la población peruana aún tiene conceptos equívocos y baja aceptación a los medicamentos genéricos. El presente estudio debería servir para desarrollar politicas de salud, que velen por el bajo costo y calidad a la hora de escoger un medicamento.

Utilización de Medicamentos; Conocimientos, Actitudes y Práctica en Salud; Comercialización de Medicamentos

\author{
Correspondencia \\ G. Mendoza-Chuctaya \\ Escuela Profesional de Medicina Humana, Universidad \\ Nacional de San Antonio Abad del Cusco. \\ APV Praderas del Inca B-5, San Sebastián Cusco / Perú. \\ giustonmch@gmail.com \\ 1 Escuela Profesional de Medicina Humana, Universidad \\ Nacional de San Antonio Abad del Cusco, Cusco, Perú. \\ 2 Servicio de Cirugía de Tórax y Cardiovascular, Hospital \\ Nacional Edgardo Rebagliati Martins, Lima, Perú. \\ 3 Facultad de Medicina Humana, Universidad Continental, \\ Huancayo, Perú. \\ 4 Facultad de Medicina "Alberto Hurtado", Universidad Peruana \\ Cayetano Heredia, Lima, Perú. \\ 5 Facultad de Medicina, Universidad de San Martín de Porres, \\ Chiclayo, Perú. \\ 6 Facultad de Medicina Humana, Universidad Nacional del \\ Altiplano, Puno, Perú.
}




\section{Introducción}

En el mundo, la industria farmacéutica ha generado un incremento de ventas, siendo las más representativas las procedentes de Estados Unidos, Francia y Alemania 1. El Perú no estuvo exento de este incremento, la Asociación Nacional de Laboratorios Farmacéuticos (ALAFARPE) reportó en el año 2017, un valor de mercado de casi USD 1.900 millones en la venta de medicamentos 2, siendo alrededor de $43-47 \%$ el gasto en salud de una familia promedio 3. Un importante subgrupo de este mercado está conformado por los medicamentos genéricos, que contienen un mismo principio activo y la misma dosis que el medicamento de marca, aunque puedan diferir en sus excipientes 4,5 . Los medicamentos de marca se diferencian de los medicamentos genéricos debido a que su patente aún se encuentra vigente, en promedio 20 años; finalizado este periodo cualquier laboratorio puede fabricarlo y comercializarlo 6,7,8. Una diferencia importante es el costo, siendo los medicamentos genéricos los que presentan un costo de venta más bajo que los de marca ${ }^{9}$. En el Perú, se establece como medicamentos genéricos el producto farmacéutico cuyo nombre corresponde a la Denominación Común Internacional (DCI) del principio activo, que no es identificado con nombre de marca; y el medicamentos de marca como el producto farmacéutico que se comercializa bajo un nombre determinado por el propio fabricante 10.

El Perú, durante los últimos 50 años ha experimentado una serie de cambios que repercutieron en el ámbito de la salud y acceso a medicamentos 11,12. En el año 1990, el Ministerio de Salud del Perú (MINSA) inició un proceso de reorganización y con el Decreto Legislativo no 584 se creó la Dirección General de Medicamentos, Insumos y Drogas (DIGEMID), que tiene como función el control de los procesos de producción, distribución y dispensación de los medicamentos en el Perú 13. En el año 1997 se promulgó la Ley General de Salud (Ley no 26842) 10, la cual institucionalizó el derecho ciudadano al libre acceso a prestaciones de salud, además, incluyó un acápite donde indica que la prescripción de medicamentos debe consignar obligatoriamente su DCI, el nombre de marca si lo tuviere, la forma farmacéutica, posología, dosis y periodo de administración 14. Años más tarde, en el 2009 se promulgó la Ley de los Productos Farmacéuticos, Dispositivos Médicos y Productos Sanitarios (Ley no 29459), donde el estado promueve el acceso universal a los productos farmacéuticos esenciales como componente fundamental de la atención integral de salud 14.

Los medicamentos genéricos han demostrado ser mucho más rentables sobre los medicamentos de marca en diversas enfermedades 15 , y al ser usados adecuadamente generan un importante ahorro en la salud pública, reduciendo los gastos sanitarios en general 16,17. En países europeos como España, el comercio de medicamentos genéricos genera un ahorro de EUR 1.000 millones anuales, lo que supone una media de EUR 50 de ahorro por hogar 18,19. Además, el conocimiento y la confianza del paciente están relacionados con el mayor uso de medicamentos genéricos en países desarrollados 20; mientras que otras investigaciones mostraron que la mayoría no identifican ni definen correctamente el concepto de medicamentos genéricos y de marca 21; asimismo, un estudio realizado en Brasil concluyó que el $30 \%$ de su población estuvo de acuerdo con que los medicamentos genéricos son menos eficaces que los de marca 22, y otro estudio en España determinó que el 56\% considera que la única diferencia entre un genérico y uno de marca es el precio 23.

En el Perú el sistema de salud está conformado por los subsectores público y privado, donde el subsector público está representado por el seguro del MINSA, el Seguro de la Seguridad Social (EsSalud), las Sanidades de Fuerzas Armadas y Policiales. Por otro lado, el subsector privado con las entidades prestadoras de salud, aseguradoras privadas, clínicas y organizaciones no gubernamentales 24 . El $76,5 \%$ de la población peruana tiene algún tipo de seguro (sea público o privado) 25 que les permite acceder a un servicio de calidad, garantizando intervenciones para diversas condiciones de salud y enfermedad 26; y a pesar de que cada año aumenta la cobertura de asegurados, el Perú sigue siendo un país en vías de desarrollo que tiene bajos recursos, múltiples problemas en salud y con una la pobreza monetaria que afecta al $21,7 \%$ de la población 27 . Por tal razón el presente estudio abarca un tema muy relevante que podría ayudar en el proceso de implementación de una política de medicamentos genéricos, al abordar la dimensión de su aceptabilidad por parte de la población. Por lo que el objetivo de la investigación fue el conocer la percepción de la población con respecto a medicamentos genéricos, frente a los de marca, en hospitales del Perú. 


\section{Material y métodos}

Estudio transversal, realizado entre los meses de febrero y marzo del año 2016, en salas de espera de 26 hospitales de 13 ciudades del Perú. La población que se tomó en cuenta residía en las ciudades de Lima, Piura, Cusco, Puno, Huancayo, Arequipa, Chiclayo, Huaraz, Huánuco, Tarapoto, Huamanga, Tacna y Cerro de Pasco (Figura 1).

El estudio incluyó a personas mayores de 18 años que eran atendidas en alguno de los 26 hospitales sedes, y que aceptaron participar en la investigación, se excluyó a los pacientes que no hablaran español o que tuviesen alguna discapacidad cognitiva (menos del 5\% de exclusión). Se utilizó un muestreo por conveniencia, usando los distintos hospitales de cada ciudad de estudio, que fueron escogidas por la posible colaboración de estudiantes de medicina en cada una de las sedes (pertenecientes a distintas sociedades científicas y que se ofrecieron para la recolección de datos de forma aleatoria). Asimismo, se calculó el tamaño muestral con un nivel de confianza de 95\%, una mínima diferencia de proporciones del $3 \%$ (60\% vs. $57 \%$ para el mínimo cruce de variables; obtenida en un piloto previo) y una potencia del $85 \%$; con todo esto se calculó que se requería 4.909 encuestados entre todas las sedes.

Para la elaboración de la encuesta se usaron preguntas propuestas por un estudio previo 22, dicho instrumento fue revisado y sometido a un proceso de evaluación, mediante una prueba piloto de 30 encuestas en salas de espera de los hospitales, donde se realizó el estudio. Se escogió este lugar de encuesta, pues era de mayor accesibilidad para los recolectores de datos, ya que estos en su totalidad eran estudiantes de medicina que cursaban con rotaciones hospitalarias. La variable dependiente que midió la percepción de eficacia de los medicamentos fue recolectada a través de la premisa: "los medicamentos genéricos son menos eficaces que los medicamentos de marca”; además, se usó otras afirmaciones como: "Ha recomendado o recomendaría usted a otras personas el uso de medicamentos genéricos", "Los médicos prefieren recetar medicamentos genéricos", "Los medicamentos de marca demoran más tiempo en tener efecto, en comparación con los medicamentos genéricos", "Los medicamentos genéricos son más adecuados o apropiados que los medicamentos de marca para enfermedades leves, banales o menos graves", "Los medicamentos de marca causan más efectos secundarios (reacciones adversas) que los medicamentos genéricos", y "Si no hubiera una diferencia de precio (los genéricos son más baratos), yo siempre prefiero tomar un medicamento de marca”.

Otra variable secundaria fue "si en los últimos tres meses había consumido algún medicamento", además, se recolectaron variables sociodemográficas y socioeconómicas como: género, edad, ingreso mensual familiar (en Soles - PEN), estado civil, nivel educativo, la dependencia del establecimiento de salud en: Ministerio de Salud (MINSA) y la seguridad social (EsSalud), y el lugar de residencia que para el análisis se dividió según tamaño poblacional en: Lima (capital del Perú con nueve millones de habitantes), grandes ciudades (más de un millón de habitantes): Piura, Cusco, Puno, Huancayo, Arequipa, Chiclayo y Huaraz; y pequeñas ciudades (menos de un millón de habitantes): Huánuco, Tarapoto, Huamanga, Tacna y Cerro de Pasco.

El proyecto de investigación fue aprobado por el comité de ética del Hospital Madre-Niño San Bartolomé (RCEI-40), ubicado en la ciudad de Lima. Se encuestó a personas que aceptaron participar voluntariamente, se les detalló el objetivo del estudio y se comunicó que su participación era voluntaria, asegurando el anonimato y confidencialidad de los datos.

En el análisis descriptivo se puso la frecuencia y porcentaje de las variables categóricas, así como, la mejor medida de tendencia central y dispersión de las variables cuantitativas. El análisis multivariado comparó las percepciones que tenían los pacientes sobre los medicamentos genéricos, según las variables secundarias, esto debido a diferencias culturales. Para la presentación descriptiva se utilizaron frecuencias absolutas y relativas, para el cruce de variables se utilizó la prueba estadística del chi cuadrado (se consideró a los valores de $\mathrm{p}<0,05$ como estadísticamente significativos). También se calcularon las razones de prevalencias (RP) y sus intervalos del 95\% de confianza (IC95\%); se usaron regresiones de Poisson crudas y ajustadas con varianza robusta (debido a que se ha mostrado que los modelos de Poisson son adecuados para estudios transversales analíticos 28), para la construcción del modelo primero se construyó un cruce bivariado, según el resultado de cada cruce las variables significativas (en esta etapa se consideró un nivel de significancia cuando se obtenía los valores de $\mathrm{p}$ $>0,05)$ fueron incluidas en el modelo final (para la selección del modelo se realizó la obtención de los resultados de forma bivariados, según la mayor significancia (y si la significancia era similar, se toma- 


\section{Figura 1}

Mapa de las 13 ciudades del Perú donde se realizó el estudio.
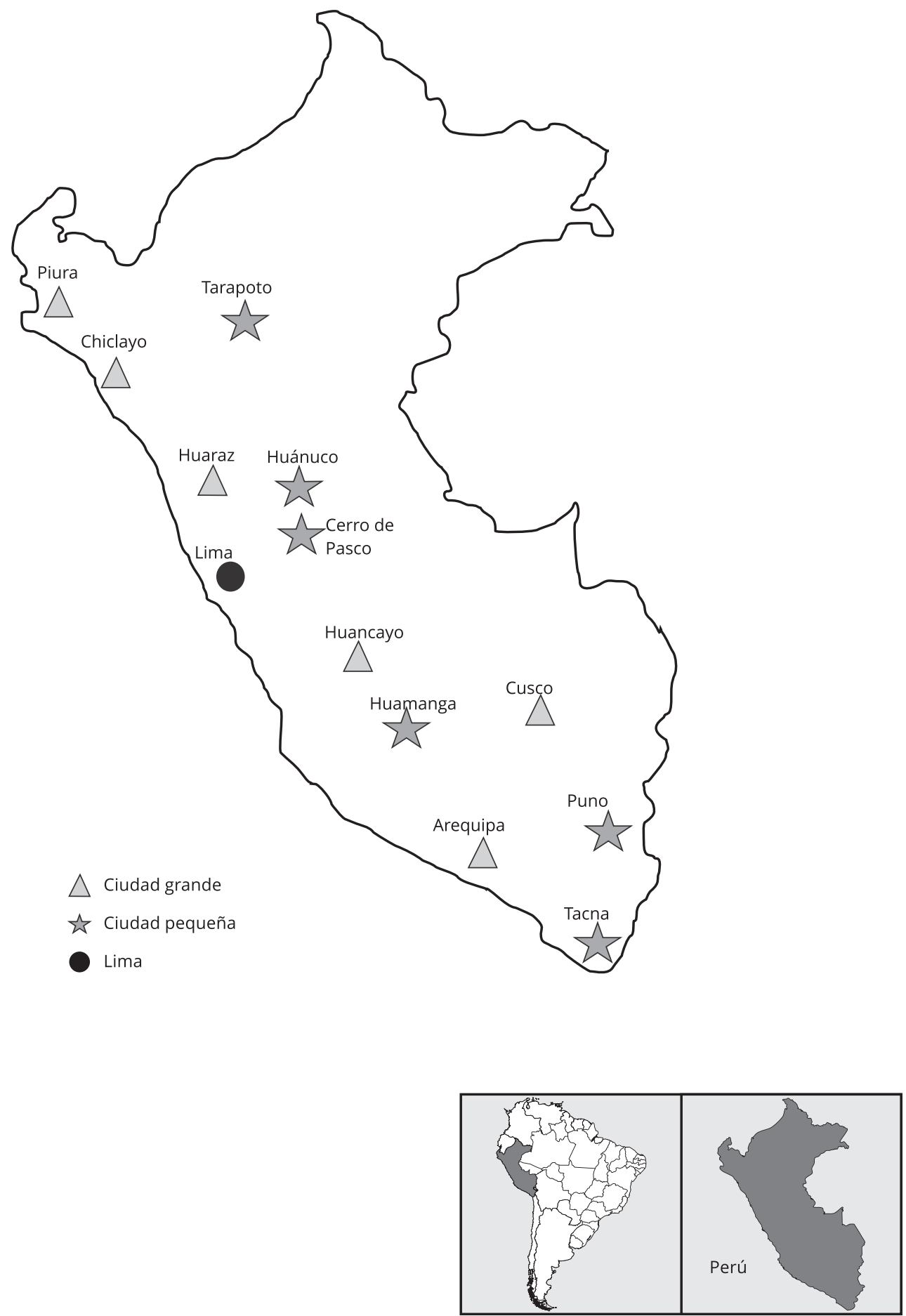
ron los valores AIC) se fue incorporando al modelo final, así hasta conseguir el resultado mostrado). De esta manera, se construyó un modelo ajustado que incluyó todas las variables socioeconómicas, demográficas, si tomaba medicamentos y sus percepciones. El análisis de los datos de realizó con el software estadístico Stata, versión 14.0 (https://www.stata.com).

\section{Resultados}

Se encuestó a 4.914 participantes de 13 ciudades del Perú, dividido en tres sectores, Lima 7,6\%, grandes ciudades $54,6 \%$, y pequeñas ciudades $37,6 \%$. Los encuestados tuvieron una edad media de $40,9 \pm$ 15,4 años; 58,4\% eran mujeres; según la dependencia del servicio de salud el 51,7\% estaban siendo atendidos en hospitales del MINSA; y según el ingreso familiar mensual 50,8\% ganaban entre PEN 1.000-4.000, características que se detallan en la Tabla 1.

Acerca de las percepciones: el 46,7\% estaban de acuerdo y el 39\% en desacuerdo con que los medicamentos genéricos son menos eficaces que los medicamentos de marca; el 38,8\% estaban de acuerdo y el 38,3\% en desacuerdo con que los medicamentos de marca causan más efectos secundarios (reacciones adversas) que los medicamentos genéricos; el 21,5\% estaban de acuerdo y el 59,5\% en

Tabla 1

Características sociodemográficas de los participantes encuestados en 26 hospitales del Perú.

\begin{tabular}{|c|c|c|c|c|c|}
\hline Variable & $\begin{array}{l}\text { Lima } \\
\text { n (\%) }\end{array}$ & $\begin{array}{c}\text { Grandes ciudades } \\
\text { n (\%) }\end{array}$ & $\begin{array}{l}\text { Pequeñas ciudades } \\
\text { n (\%) }\end{array}$ & $\begin{array}{l}\text { Total } \\
\text { n (\%) }\end{array}$ & Valor de $p$ * \\
\hline Edad [média \pm DE] & $48,6 \pm 18$ & $39,8 \pm 15,4$ & $41 \pm 14,4$ & $40,9 \pm 15,4$ & \\
\hline Edad en terciles (años) & & & & & $<0,001$ \\
\hline $18-32$ & $80(21,2)$ & $1.019(38,0)$ & $552(29,8)$ & $1.651(33,6)$ & \\
\hline $33-47$ & $93(24,6)$ & $847(31,5)$ & $730(39,4)$ & $1.670(34,0)$ & \\
\hline $48-97$ & $205(54,2)$ & $818(30,5)$ & $570(30,8)$ & $1.593(32,4)$ & \\
\hline Sexo & & & & & $<0,001$ \\
\hline Masculino & $144(38,1)$ & $1.044(38,9)$ & $857(46,3)$ & $2.045(41,6)$ & \\
\hline Femenino & $234(61,9)$ & $1.640(61,1)$ & $995(53,7)$ & $2.869(58,4)$ & \\
\hline Con pareja & & & & & 0,003 \\
\hline No & $156(41,3)$ & $1.147(42,7)$ & $631(34,1)$ & $1.934(39,4)$ & \\
\hline Sí & $222(58,7)$ & $1.537(57,3)$ & $1221(65,9)$ & $2.980(60,6)$ & \\
\hline Ingresos familiares (PEN) & & & & & $<0,001$ \\
\hline Mayor a 4.000 & $15(4,0)$ & $292(10,9)$ & $182(9,8)$ & $489(10,0)$ & \\
\hline $1.000-4.000$ & $186(49,2)$ & $1.168(43,5)$ & $1143(61,7)$ & $2.497(50,8)$ & \\
\hline Menos de 1.000 & $177(46,8)$ & $1.224(45,6)$ & $527(28,5)$ & $1.928(39,2)$ & \\
\hline Nivel educativo & & & & & $<0,001$ \\
\hline Primaria & $67(17,1)$ & $321(12,1)$ & $153(8,2)$ & $541(11,0)$ & \\
\hline Secundaria & $152(38,8)$ & $849(31,8)$ & $840(45,2)$ & $1.841(37,5)$ & \\
\hline Superior & $172(43,9)$ & $1.495(56,1)$ & $865(46,5)$ & $2.532(51,5)$ & \\
\hline $\begin{array}{l}\text { Actualmente o hace } 3 \text { meses ha tomado un } \\
\text { medicamento }\end{array}$ & & & & & $<0,001$ \\
\hline Sí & $248(65,6)$ & $1.656(61,7)$ & $1337(72,2)$ & $3.241(66,0)$ & \\
\hline No & $130(34,4)$ & $1.028(38,3)$ & $515(27,8)$ & $1.673(34,0)$ & \\
\hline Dependencia de salud & & & & & 0,084 \\
\hline MINSA & $189(50,0)$ & $1.424(53,1)$ & $928(50,1)$ & $2.541(51,7)$ & \\
\hline EsSalud & $189(50,0)$ & $1.260(46,9)$ & $924(49,9)$ & $2.373(48,3)$ & \\
\hline
\end{tabular}

DE: desviación estándar; EsSalud: Seguro de la Seguridad Social; MINSA: Ministerio de Salud del Perú.

* Los valores de $p$ fueron obtenidos con la prueba del chi cuadrado. 
desacuerdo con que los medicamentos de marca demoran más tiempo en tener efecto, en comparación con los medicamentos genéricos; el 40,4\% estaban de acuerdo y el 41,3\% en desacuerdo con que los medicamentos genéricos son más adecuados o apropiados que los medicamentos de marca para enfermedades leves, banales o menos graves; el 48,7\% estaban de acuerdo y el 33,7\% en desacuerdo con que los médicos prefieren recetar medicamentos genéricos; el 59,7\% estaban de acuerdo y el $30,9 \%$ en desacuerdo con que si no hubiera una diferencia de precio (los medicamentos genéricos son más baratos), prefieren tomar un medicamentos de marca; el 49,3\% estaban de acuerdo y el 30\% en desacuerdo con que ha recomendado o recomendaría a otras personas el uso de medicamentos genéricos. Se analizó también la percepción según el lugar de residencia, especificándose en la Tabla 2.

En el análisis multivariado se encontró que las personas que tenían un ingreso económico menor a PEN 1.000 estaban predispuestas a recomendar un medicamento genérico (RP = 1,36; IC95\%: 1,141,63); los participantes que residen en pequeñas ciudades preferían tomar un medicamento de marca, si no hubiera diferencias en precios ( $\mathrm{RP}=1,35$; IC95\%: 1,17-1,56); además, los participantes con un nivel educativo superior afirmaban que los médicos prefieren recetar medicamentos genéricos $(\mathrm{RP}=$ 1,24; IC95\%: 1,05-1,46) (Tabla 3).

\section{Discusión}

Este estudio peruano evaluó las percepciones sobre los medicamentos, donde el 46,7\% de los participantes cree que los medicamentos de marca son más eficaces que los medicamentos genéricos, este resultado supera a otros estudios, como el realizado en Brasil, donde el $30 \%$ estuvo de acuerdo con que los medicamentos genéricos son menos eficaces que los de marca 22, en Irlanda, el 24\% de los encuestados cree que los medicamentos genéricos son de menos calidad que los medicamentos de marca 29 , y en el sureste de Estados Unidos el 23\% cree que los medicamentos de marca son más efectivos que los medicamentos genéricos 30 . Esto demuestra que gran parte de la población peruana percibe que los medicamentos de marca son más eficaces, hecho que podría influenciar el incremento de compra de estos, aumentando y contribuyendo indirectamente el gasto en salud.

Además, seis de cada diez encuestados están de acuerdo en tomar un medicamentos de marca si no existiera diferencia en el precio con un medicamentos genéricos, y no están de acuerdo en que los medicamentos de marca demoran más tiempo en tener efecto, en comparación con los medicamentos genéricos; estos resultados ponen en manifiesto que la mayor parte de la población tiene conceptos equívocos sobre los medicamentos genéricos, esta falta de información podría estar influenciada por el nivel educativo de los participantes, ya que varios estudios han demostrado que a mayor educación, aumenta la aceptación sobre los medicamentos genéricos 31,32 , el uso correcto de los mismos 33,34, y disipa los mitos sobre sus diferencias 16 . Sin embargo, el análisis multivariado de nuestro estudio al momento de afirmar si los medicamentos genéricos eran menos eficaces que los medicamentos de marca, en los participantes con un nivel educativo superior, resultó no significativo.

El 49,3\% de los participantes han recomendado o recomendarían a otras personas el uso de medicamentos genéricos, además, el análisis multivariado señaló este hecho en aquellos con ganancias menores a PEN 1.000, mostrando preferencias por los participantes con menores ingresos económicos; caso similar descrito en un trabajo realizado en el Perú, donde la mayor parte de la población de clase social media tuvo una opinión buena a regular acerca de los medicamentos genéricos 24 . A pesar de que en otros estudios los participantes con un mayor ingreso económico prefieren tomar un medicamentos genéricos que uno de marca 35; este hecho podría ser explicado, ya que el Perú siendo un país en vías de desarrollo las personas que tienen menor ingreso económico viven en pequeñas ciudades, y tienden a adquirir medicamentos de menor coste en relación a sus posibilidades económicas, y no necesariamente tener un grado de confianza sobre estos.

El 48,7\% la población encuestada está de acuerdo con que los médicos prefieren recetar medicamentos genéricos, este resultado podría estar influenciado, pues la recolección de datos fue realizada en hospitales públicos, donde los pacientes en su mayoría cuentan con algún tipo de seguro; en contraparte, la otra mitad no estarían de acuerdo con que los médicos prefieren recetar medicamentos genéricos. En relación a estos hallazgos, se deben realizar investigaciones que traten de explicar por qué algunos médicos no recetan medicamentos genéricos, ya que, según establece la ley peruana existe la 
Tabla 2

Percepción de medicamentos genéricos frente a los de marca, según lugar de residencia en pacientes encuestados en 26 hospitales del Perú.

\begin{tabular}{|c|c|c|c|c|c|}
\hline $\begin{array}{l}\text { Percepciones sobre los medicamentos de marca y } \\
\text { genéricos }\end{array}$ & $\begin{array}{l}\text { Lima } \\
\text { n (\%) }\end{array}$ & $\begin{array}{l}\text { Grandes ciudades } \\
\qquad \mathbf{n}(\%)\end{array}$ & $\begin{array}{l}\text { Pequeñas ciudades } \\
\text { n (\%) }\end{array}$ & $\begin{array}{l}\text { Total } \\
\text { n (\%) }\end{array}$ & Valor de $p$ \\
\hline $\begin{array}{l}\text { Los medicamentos genéricos son menos eficaces que los } \\
\text { de marca }\end{array}$ & & & & & $<0,001$ \\
\hline De acuerdo & $187(49,5)$ & $1.293(48,2)$ & $816(44,1)$ & $2.296(46,7)$ & \\
\hline En desacuerdo & $161(42,6)$ & $1.060(39,5)$ & $697(37,6)$ & $1.918(39,0)$ & \\
\hline No sabe & $30(7,9)$ & $331(12,3)$ & $339(18,3)$ & $700(14,3)$ & \\
\hline $\begin{array}{l}\text { Los medicamentos de marca causan más efectos } \\
\text { secundarios (reacciones adversas) que los medicamentos } \\
\text { genéricos }\end{array}$ & & & & & 0,005 \\
\hline De acuerdo & $140(37,0)$ & $1.057(39,4)$ & $711(38,4)$ & $1.908(38,8)$ & \\
\hline En desacuerdo & $167(44,2)$ & $1.046(39,0)$ & $667(36,0)$ & $1.880(38,3)$ & \\
\hline No sabe & $71(18,8)$ & $581(21,7)$ & $474(25,6)$ & $1.126(22,9)$ & \\
\hline $\begin{array}{l}\text { Los medicamentos de marca demoran más tiempo en } \\
\text { tener efecto, en comparación con los medicamentos } \\
\text { genéricos }\end{array}$ & & & & & $<0,001$ \\
\hline De acuerdo & $42(11,1)$ & $523(19,5)$ & $493(26,6)$ & $1.058(21,5)$ & \\
\hline En desacuerdo & $305(80,7)$ & $1.747(65,1)$ & $874(47,2)$ & $2.926(59,5)$ & \\
\hline No sabe & $31(8,2)$ & $414(15,4)$ & $485(26,2)$ & $930(18,9)$ & \\
\hline $\begin{array}{l}\text { Los medicamentos genéricos son más adecuados o } \\
\text { apropiados que los medicamentos de marca para } \\
\text { enfermedades leves, banales o menos graves }\end{array}$ & & & & & $<0,001$ \\
\hline De acuerdo & $221(58,5)$ & $1.014(37,8)$ & $750(40,5)$ & $1.985(40,4)$ & \\
\hline En desacuerdo & $118(31,2)$ & $1.267(47,2)$ & $642(34,7)$ & $2.027(41,3)$ & \\
\hline No sabe & $39(10,3)$ & $403(15,0)$ & $460(24,8)$ & $902(18,4)$ & \\
\hline Los médicos prefieren recetar medicamentos genéricos & & & & & $<0,001$ \\
\hline De acuerdo & $216(57,1)$ & $1.358(50,6)$ & $817(44,1)$ & $2.391(48,7)$ & \\
\hline En desacuerdo & $113(29,9)$ & $988(36,8)$ & $556(30,0)$ & $1.657(33,7)$ & \\
\hline No sabe & $49(13,0)$ & $338(12,6)$ & $479(25,9)$ & $866(17,6)$ & \\
\hline $\begin{array}{l}\text { Si no hubiera una diferencia de precio (los genéricos son } \\
\text { más baratos), yo siempre prefiero tomar un medicamento } \\
\text { de marca }\end{array}$ & & & & & $<0,001$ \\
\hline De acuerdo & $279(73,8)$ & $1.625(60,5)$ & $1.027(55,5)$ & $2.931(59,7)$ & \\
\hline En desacuerdo & $80(21,2)$ & $779(29,0)$ & $659(35,6)$ & $1.518(30,9)$ & \\
\hline No sabe & $19(5,0)$ & $280(10,4)$ & $166(9,0)$ & $465(9,5)$ & \\
\hline $\begin{array}{l}\text { Ha recomendado o recomendaría usted a otras personas } \\
\text { el uso de medicamentos genéricos }\end{array}$ & & & & & $<0,001$ \\
\hline Sí lo recomendaría & $220(58,2)$ & $1.508(56,2)$ & $695(37,5)$ & $2.423(49,3)$ & \\
\hline No lo recomendaría & $130(34,4)$ & $698(26,0)$ & $646(34,9)$ & $1.474(30,0)$ & \\
\hline No sabe & $28(7,4)$ & $478(17,8)$ & $511(27,6)$ & $1.017(20,7)$ & \\
\hline
\end{tabular}

obligación de prescribir medicamentos en su DCI, y poder escribir en la receta el nombre de marca si lo tuviera 10,36, siendo esta un tipo de estrategia recomendada para un mejor uso de medicamentos 37 .

El presente estudio refleja una encuesta de percepción sobre los medicamentos, y no un análisis de recetas médicas, además, estuvo sujeta a sesgo de información, debido a que no se pudo controlar algunas ocurrencias que pudieran haber alterado las percepciones de los medicamentos genéricos o de marca (no se tuvo información acerca de las marcas de los fármacos que utilizaron anteriormente, si alguno hubiese estado adulterado, vencido o si pudo haber ocasionado alguna reacción adversa). 
Tabla 3

Análisis multivariado de la percepción de medicamentos genéricos frente a los de marca en pacientes encuestados en 26 hospitales del Perú.

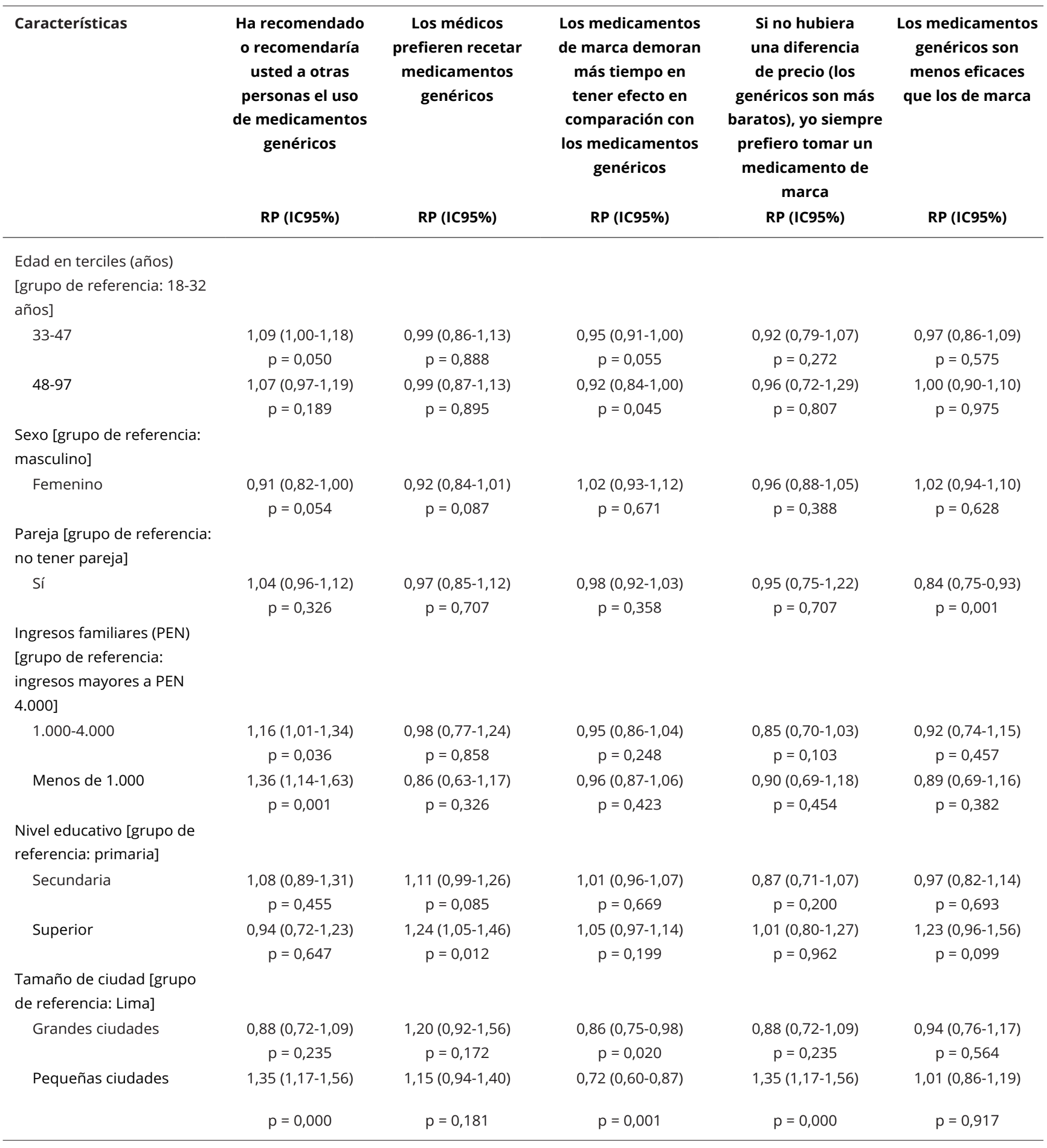

(continúa) 
Tabla 3 (continuación)

\begin{tabular}{|c|c|c|c|c|c|}
\hline Características & $\begin{array}{l}\text { Ha recomendado } \\
\text { o recomendaría } \\
\text { usted a otras } \\
\text { personas el uso } \\
\text { de medicamentos } \\
\text { genéricos }\end{array}$ & $\begin{array}{l}\text { Los médicos } \\
\text { prefieren recetar } \\
\text { medicamentos } \\
\text { genéricos }\end{array}$ & $\begin{array}{c}\text { Los medicamentos } \\
\text { de marca demoran } \\
\text { más tiempo en } \\
\text { tener efecto en } \\
\text { comparación con } \\
\text { los medicamentos } \\
\text { genéricos }\end{array}$ & $\begin{array}{c}\text { Si no hubiera } \\
\text { una diferencia } \\
\text { de precio (los } \\
\text { genéricos son más } \\
\text { baratos), yo siempre } \\
\text { prefiero tomar un } \\
\text { medicamento de } \\
\text { marca }\end{array}$ & $\begin{array}{l}\text { Los medicamentos } \\
\text { genéricos son } \\
\text { menos eficaces } \\
\text { que los de marca }\end{array}$ \\
\hline & RP (IC95\%) & RP (IC95\%) & RP (IC95\%) & RP (IC95\%) & RP (IC95\%) \\
\hline \multicolumn{6}{|l|}{$\begin{array}{l}\text { Actualmente o hace } 3 \\
\text { meses ha tomado un } \\
\text { medicamento [grupo de } \\
\text { referencia: si dice haber } \\
\text { tomado un medicamento] }\end{array}$} \\
\hline No & $\begin{array}{c}1,06(0,97-1,15) \\
p=0,203\end{array}$ & $\begin{array}{c}1,11(0,99-1,24) \\
p=0,080\end{array}$ & $\begin{array}{c}0,99(0,93-1,05) \\
p=0,726\end{array}$ & $\begin{array}{c}0,94(0,75-1,18) \\
p=0,590\end{array}$ & $\begin{array}{c}1,08(0,94-1,24) \\
p=0,298\end{array}$ \\
\hline \multicolumn{6}{|l|}{$\begin{array}{l}\text { Dependencia de salud } \\
\text { [grupo de referencia: } \\
\text { MINSA] }\end{array}$} \\
\hline EsSalud & $\begin{array}{c}1,21(1,08-1,37) \\
p=0,002\end{array}$ & $\begin{array}{c}0,94(0,76-1,16) \\
p=0,544\end{array}$ & $\begin{array}{c}1,03(0,93-1,14) \\
p=0,595\end{array}$ & $\begin{array}{c}0,88(0,64-1,20) \\
p=0,420\end{array}$ & $\begin{array}{c}0,96(0,85-1,08) \\
p=0,505\end{array}$ \\
\hline
\end{tabular}

IC95\%: intervalo del 95\% de confianza; EsSalud: Seguro de la Seguridad Social; MINSA: Ministerio de Salud del Perú; RP: razón de prevalencia. Nota: se utilizaron regresiones de Poisson crudas y ajustadas con varianza robusta; para la construcción del modelo primero se construyó un cruce bivariado, según el resultado de cada cruce las variables significativas (en esta etapa se consideró un nivel de significancia cuando se obtenía los valores de $p>0,05$ ) fueron incluidas en el modelo final (para la selección del modelo se realizó la obtención de los resultados de forma bivariados, según la mayor significancia se fue incorporando al modelo final, así hasta conseguir el resultado mostrado).

Puesto que los consumidores y médicos saben menos que el fabricante acerca de la real eficacia del medicamento, y estas circunstancias pudieron haber influido en la percepción de cada participante. Se recomienda que las instituciones encargadas de velar por la calidad de los medicamentos, como DIGEMID, promueva y genere investigaciones que puedan ajustar estos factores, ya que ellos son los encargados de la vigilancia en aspectos relacionados con la calidad, seguridad y eficacia de los medicamentos en el Perú 13, para así poder desarrollar políticas de salud que velen por la población a la hora de escoger entre un medicamento genérico o de marca.

A pesar de que la cobertura en salud se ha incrementado con múltiples estrategias, lo que se ha reflejado en más atenciones preventivas, satisfacción de los asegurados y reducción del gasto de bolsillo en medicamentos 38 , aún quedan desafíos en el sistema de salud peruano, ya que, al tener una población, donde la mayoría no tiene un gran poder adquisitivo, el presente estudio debería apoyar en la generación de estrategias para la promoción del uso adecuado de los medicamentos genéricos, e incrementar la aceptabilidad para un mayor uso de estos. La Ley de los Productos Farmacéuticos, Dispositivos Médicos y Productos Sanitarios (Ley no 29459), promueve el acceso universal a los productos farmacéuticos, además de la obligación de mantener reservas mínimas de productos farmacéuticos esenciales disponibles en farmacias de hospitales públicos, de acuerdo a su nivel de complejidad y población en general (Art. 27) 39. En la actualidad, en el Perú se viene discutiendo iniciativas legislativas que pretenden modificar el Art. 27 de la Ley $n^{\circ}$ 29459, que busca fomentar la oferta de medicamentos genéricos no solo en farmacias públicas, sino también en privadas, esta iniciativa legislativa podría ayudar a la aceptabilidad de los medicamentos genéricos por parte de la población peruana.

Por otro lado, hay poco protagonismo de la DIGEMID al no contar con una organización, ni recursos suficientes para exigir el cumplimiento de las normativas vigentes en todos los establecimientos farmacéuticos 9,40. Por lo que se recomienda al MINSA y a las instituciones rectoras de 
salud que informen, potencien y realicen intervenciones para incrementar el uso de medicamentos genéricos. Asimismo, alentar a la realización de mayores investigaciones y la inversión de productos genéricos de buena calidad, ya que, existen laboratorios especializados de dichos productos que hacen una competencia directa por su alta calidad y bajo costo de los fármacos 37 , así como también trabajos de intervención en la población 41 y desarrollar políticas de salud que velen por la correcta elección entre un medicamento genérico o de marca, como lo hacen otros países 8,42,43.

\section{Conclusión}

En nuestro estudio se encontró que casi la mitad de los participantes creen que los medicamentos genéricos son menos eficaces que los medicamentos de marca, resultado que pone en manifiesto que la población peruana aún tiene conceptos equívocos y baja aceptación a los medicamentos genéricos. Asimismo, los participantes que tenían un menor ingreso económico estaban más predispuestos a recomendar un medicamento genérico, indicando quizá un grado de confianza, o que solo son adquiridos en relación a sus posibilidades económicas. Y a pesar de que varios estudios demuestran que a mayor educación aumenta la aceptación, y el uso correcto de los medicamentos genéricos, en nuestro estudio dicha variable resultó no significativa.

\section{Colaboradores}

G. Mendoza-Chuctaya y J. E. Chachaima-Mar concibieron y diseñaron el proyecto de investigación, recolectaron los datos, realizaron el análisis estadístico, redactaron el manuscrito y aprobaron su versión final. W. S. Cubas-Llalle, C. R. Mejía, R. Montesinos-Segura y J. C. Mamani-Cruz recolectaron los datos, realizaron el análisis estadístico, redactaron el manuscrito y aprobaron su versión final. L. R. Arce-Villalobos realizó el análisis estadístico, redactó el manuscrito y aprobó su versión final.

\section{Informaciones adicionales}

ORCID: Giuston Mendoza-Chuctaya (0000-00025389-5640); Wildor Samir Cubas-Llalle (00000002-5380-7372); Christian R. Mejía (0000-00025940-7281); Jorge Emerson Chachaima-Mar (00000002-5862-5295); Reneé Montesinos-Segura (0000-0001-5426-830X); Laura R. Arce-Villalobos (0000-0001-8675-4177); John Carlos MamaniCruz (0000-0003-2838-0626).

\section{Agradecimientos}

La presente investigación agradece a los estudiantes de Medicina: Junior David Herna Paiva, Keyla Estefani Ramos Ramirez, Melissa Claudia Llaiqui Condori, Lucciano Gambetta Meléndez, Marivel Liz Castro Ortega, Isabel Patricia Egoavil Araujo, Yarlyn Jemmy Nahidú Velasquez Cabrera, Karla García Perez, Victor Roman Lazarte quienes participaron activamente en la recolección de datos.

\section{Referencias}

1. Global Healthcare Trends in 2017. https:// www.bmiresearch.com/events/global-healthcare-trends-in-2017 (accedido el 15/Jul/2018).

2. Alafarpe: laboratorios ven oportunidades de participar en el mecanismo de Obras por Impuestos. Gestión 2017; 11 ago. https://gestion. pe/economia/empresas/alafarpe-laboratoriosven-oportunidades-participar-mecanismoobras-impuestos-141319.

3. Petrera M. Reflexiones para la política sectorial de salud en Perú a partir de las Cuentas Nacionales de Salud. Economía 2016; 39:3565.

4. European Medicines Agency. Guideline on the investigation of bioequivalence. https://www. ema.europa.eu/en/documents/scientificguideline/guideline-investigation-bioequiva lence-rev1_en.pdf (accedido el 13/Dic/2017).

5. Garcia Arieta A, Hernández Garcia C, Avendaño Sóla C. Regulación de los medicamentos genéricos: evidencias y mitos. Inf Ter Sist Nac Salud 2010; 34:71-82.

6. Pike J, Brown A, Becker S. Barreras de entrada a los medicamentos genéricos en la Unión Europea. Madrid: Asociación Española de Fabricantes de Sustancias y Especialidades Farmacéuticas Genéricas; 2008.

7. Díez MV, Errecalde MF. Aclaraciones al concepto de genérico. Inf Ter Sist Nac Salud 1998; 22:68-72.

8. Dunne S, Shannon B, Dunne C, Cullen W. A review of the differences and similarities between generic drugs and their originator counterparts, including economic benefits associated with usage of generic medicines, using Ireland as a case study. BMC Pharmacol Toxicol 2013; 14:1. 
9. Aravena V, Calero C, Martínez O, Navarro M, Villareal R. Desarrollo del medicamento genérico en el Perú. Lima: Editorial Cordillera/ Universidad ESAN; 2008.

10. Congreso de la República. Ley no 26842: Ley General de Salud. Lima: Congreso de la República; 1997.

11. Laing R, Waning B, Gray A, Ford N, 't Hoen E. 25 years of the WHO essential medicines lists: progress and challenges. Lancet 2003; 361:1723-9.

12. Romero CP. Reforma del sector salud y la política farmacéutica en Perú. Cad Saúde Pública 2002; 18:1121-38.

13. Ministerio de Salud. Decreto Legislativo no 584. http://www.digemid.minsa.gob.pe/Main. asp?Seccion=641 (accedido el 20/Nov/2018).

14. Alcalde-Rabanal JE, Lazo-González O, Nigenda G. Sistema de salud de Perú. Salud Pública Méx 2011; 53 Suppl 2:S243-54.

15. Shrank WH, Choudhry NK, Liberman JN, Brennan TA. The use of generic drugs in prevention of chronic disease is far more costeffective than thought, and may save money. Health Aff (Millwood) 2011; 30:1351-7.

16. Dunne SS, Dunne CP. What do people really think of generic medicines? A systematic review and critical appraisal of literature on stakeholder perceptions of generic drugs. BMC Med 2015; 13:173.

17. Cameron A, Mantel-Teeuwisse AK, Leufkens HG, Laing RO. Switching from originator brand medicines to generic equivalents in selected developing countries: how much could be saved. Value Health 2012; 15:664-73.

18. CINFA. Estudio sobre valoración de los medicamentos genéricos en la población española 2013. http://www.aeseg.es/documentos/Estudio_Percepcion_M_Genericos_2013.pdf (accedido el 20/Nov/2018).

19. Redpacientes. Estudio sobre la valoración de los medicamentos genéricos en la población española. http://redpacientes.com/attach ments/resumen_nota_prensa_concurso.pdf (accedido el 20/Nov/2018).

20. Hassali MAA, Shafie AA, Jamshed S, Ibrahim MI, Awaisu A. Consumers' views on generic medicines: a review of the literature. Int J Pharm Pract 2009; 17:79-88.

21. Soler RP, Colet MP, Yahni CZ, Puig AMG. Establecimiento de los conocimientos, actitudes y opiniones de la población sobre el uso racional de medicamentos. Aten Prim 2015; 47:446-55.

22. Nardi EP, Ferraz MB, Pinheiro G, Kowalski SC, Sato EI. Perceptions of the population regarding generic drugs in Brazil: a nationwide survey. BMC Public Health 2015; 15:117.

23. KantarHealth. Percepción social sobre la imagen de los medicamentos de marca frente a genéricos. http://www.redaccionmedica.com/ contenido/images/150428_Dossier de prensa. pdf (accedido el 20/Nov/2018).
24. Tizón J. Percepción del uso de genéricos en usuarios de farmacias privadas en un distrito de Lima [Tesis de Maestría]. Rio de Janeiro: Escola Nacional de Saúde Pública Sergio Arouca, Fundação Oswaldo Cruz; 2014.

25. INEI: el $76.5 \%$ de la población tiene algún seguro de salud. Gestión 2017; 20 dic. https:// gestion.pe/economia/inei-76-5-poblacionseguro-salud-223173.

26. Wilson L, Velásquez A, Ponce C. La Ley Marco de Aseguramiento Universal en Salud en el Perú: análisis de beneficios y sistematización del proceso desde su concepción hasta su promulgación. Rev Peru Med Exp Salud Pública 2009; 26:207-17.

27. Intituto Nacional de Estadística e Informática. Pobreza monetaria afectó al $21,7 \%$ de la población del país durante el año 2017. https:// www.inei.gob.pe/prensa/noticias/pobrezamonetaria-afecto-al-217-de-la-poblacion-delpais-durante-el-ano-2017-10711/ (accedido el 20/Nov/2018).

28. Farfán-García RC, Ulloque JL, Araujo-Castillo RV. Medidas de asociación en estudios transversales: a propósito del estudio "Elevada frecuencia de dislipidemia en pacientes infectados por VIH en un hospital público peruano”. Rev Peru Med Exp Salud Pública 2018; 35:163-4.

29. Dunne S, Shannon B, Dunne C, Cullen W. Patient perceptions of generic medicines: a mixed-methods study. Patient 2014; 7:177-85.

30. Keenum AJ, Devoe JE, Chisolm DJ, Wallace LS. Generic medications for you, but brand-name medications for me. Res Soc Adm Pharm 2012; 8:574-8.

31. Benjamin D, Swartz M, Forman L. The impact of evidence-based education on prescribing in a psychiatry residency. J Psychiatr Pract 2011; 17:110-7.

32. Sedjo RL, Cox ER. The influence of targeted education on medication persistence and generic substitution among consumer-directed health care enrollees. Health Serv Res 2009; 44:2079-92.

33. Quintal C, Mendes P. Underuse of generic medicines in Portugal: an empirical study on the perceptions and attitudes of patients and pharmacists. Health Policy 2012; 104:61-8.

34. Roman B. Patients' attitudes towards generic substitution of oral atypical antipsychotics: a questionnaire-based survey in a hypothetical pharmacy setting. CNS Drugs 2009; 23:693701.

35. Shrank WH, Cox ER, Fischer MA, Mehta J, Choudhry NK. Patients' perceptions of generic medications. Health Aff (Millwood) 2009; 28:546-56.

36. El Peruano: establece la obligatoriedad para los profesionales médicos de prescribir bajo Denominación Común Internacional. http:// www.digemid.minsa.gob.pe/UpLoad/UpLoa ded/PDF/LEYN26842.pdf (accedido el 20/ Nov/2018). 
37. Laing R, Hogerzeil H, Ross-Degnan D. Ten recommendations to improve use of medicines in developing countries. Health Policy Plan 2001; 16:13-20.

38. Velásquez A. Salud en el Perú: hacia la cobertura universal y una respuesta efectiva frente a riesgos sanitarios. Rev Peru Med Exp Salud Pública 2016; 33:397-8.

39. Dongo V. Ley no 29459: ley de los productos farmacéuticos, dispositivos médicos y productos sanitarios. Rev Peru Med Exp Salud Pública $2009 ; 26: 517-29$.

40. Medina E, Bel E, Suñé JM. Counterfeit medicines in Peru: a retrospective review (19972014). BMJ Open 2016; 6:e010387.
41. Vallès JA, Barreiro M, Cereza G, Ferro JJ, Martínez MJ, Escribà JM, et al. A prospective multicenter study of the effect of patient education on acceptability of generic prescribing in general practice. Health Policy 2003; 65:269-75.

42. Sharrad AK, Hassali MA. Consumer perception on generic medicines in Basrah, Iraq: preliminary findings from a qualitative study. Res Soc Adm Pharm 2011; 7:108-12.

43. Patel A, Gauld R, Norris P, Rades T. Quality of generic medicines in South Africa: perceptions versus reality - a qualitative study. BMC Health Serv Res 2012; 12:297. 
Abstract

The study aimed to investigate the population's perceptions of generic drugs compared to original brand-name drugs in Peruvian hospitals. Participation included 4,914 persons 18 years and older in 13 cities in Peru, categorized as Lima, large cities, and small cities. The study explored socioeconomic and demographic characteristics and perceptions of generics in comparison to brand-name drugs. In determining the associations for each intersection of variables, the authors calculated the prevalence ratios $(P R)$ and $95 \%$ confidence intervals (95\%CI), using crude and adjusted Poisson regression with robust variance with Stata 14.0. Of the 4,914 participants, $46.7 \%$ felt that generics are less effective than brand-name drugs and 49.3\% had recommended or would recommend generics to other people; multivariate analysis showed that individuals with income less than PEN 1,000 (USD 300) were prone to recommending a generic drug $(P R=1.36$; 95\%CI: 1.14-1.63). The results indicate that the Peruvian population still has mistaken concepts and low acceptance of generic drugs, and the study should serve to develop health policies that ensure low cost and quality when choosing medicines.

Drug Utilization; Health Knowledge, Attitudes, Practice; Pharmaceutical Trade

\section{Resumo}

O objetivo do estudo foi conhecer a percepção da população a respeito dos medicamentos genéricos frente aos medicamentos de marca em hospitais do Peru. Participaram 4.914 pessoas maiores de 18 anos, de 13 cidades do Peru; foram classificadas em Lima, além de grandes e pequenas cidades. Foram exploradas as características socioeconômicas, demográficas e de percepção de medicamentos genéricos em comparação com os medicamentos de marca. Determinando as associações para cada intersecção de variáveis, foram calculadas as razões de prevalências $(R P)$ e seus intervalos de $95 \%$ de confiança (IC95\%), usando regressões de Poisson brutas e ajustadas com variância robusta com Stata 14.0. Dos 4.914 participantes, um 46,7\% estavam de acordo com que os medicamentos genéricos são menos eficazes do que os medicamentos de marca, um 49,3\% tem recomendado o recomendaria a outras pessoas o uso de medicamentos genéricos, além disso, a análise multivariada encontrou que as pessoas que tinham um ingresso econômico menor a PEN 1.000 eram propensas a recomendar um medicamento genérico $(R P=1,36$; IC95\%: 1,14-1,63). Os resultados manifestam que a população peruana ainda tem conceitos equívocos e baixa aceitação dos medicamentos genéricos, e o presente estudo deveria servir para desenvolver politicas de saúde, que assegurem o baixo custo e a qualidade na hora de escolher um medicamento.

Uso de Medicamentos; Conhecimentos, Atitudes e Prática em Saúde; Comercialização de Medicamentos
Recibido el 29/Mar/2018

Versión final presentada el 12/Abr/2019

Aprobado el 30/Abr/2019 